\title{
PERCEPCIONES PERCEPCIONES SOBRE EL DESARROLLO DE COMPETENCIAS INVESTIGATIVAS EN ESTUDIANTES DE SEMILLEROS DE INVESTIGACIÓN
}

\section{PERCEPTIONS ON THE DEVELOPMENT OF RESEARCH COMPETENCES IN STUDENTS OF RESEARCH SEEDBEDS}

Cesar Augusto Hernández Suárez ${ }^{1}$

Audin Aloiso Gamboa Suárez ${ }^{2}$

Raúl Prada Núñez ${ }^{3}$

$4 \quad 04$

\section{RESUMEN}

Las competentes investigativas en los sujetos promueven el desarrollo individual y social, además contribuyen a la formación de habilidades cognitivas de orden superior. El presente artículo de investigación muestra los resultados de un estudio que buscó identificar las percepciones que tienen estudiantes de cinco semilleros de investigación sobre el desarrollo de competencias investigativas desde su experiencia en la universidad. El paradigma de investigación se enmarcó en el

1 Docente investigador de la Universidad Francisco de Paula Santander. Magíster en Enseñanza de las Ciencias por la Universidad Nacional Experimental del Estado Táchira (Venezuela). Correo electrónico: cesaraugusto@ ufps.edu.co, Orcid: 0000-0002-7974-5560

2 Docente investigador de la Universidad Francisco de Paula Santander. Doctor en Ciencias de la Educación por la Universidad de Cartagena. Correo electrónico: audingamboa@ufps.edu.co, Orcid: 0000-0001-9755-6408

3 Docente investigador de la Universidad Francisco de Paula Santander. Magíster en Ingeniería de Análisis de Datos, Mejora de Procesos y Toma de Decisiones por la Universidad Politécnica de Valencia (España). Correo electrónico: raulprada@ufps.edu.co, Orcid:0000-0001-6145-1786 enfoque cuantitativo - descriptivo. Se aplicó una encuesta de percepción a 82 estudiantes de cinco semilleros pertenecientes a cuatro grupos de investigación. El estudio logra revelar que gran parte de los estudiantes utilizan con mayor frecuencia las tecnologías digitales para la búsqueda de información relevante para sus trabajos de investigación y muestran un manejo de las bases de datos especializadas para esta búsqueda.

PALABRAS CLAVE: competencias investigativas, educación superior, formación en investigación.

\begin{abstract}
ABSTRAC
Competent research in subjects promote individual and social development, and also contribute to the formation of higher-order cognitive skills. This research article shows
\end{abstract}


the results of a study that sought to identify the perceptions that students from five research hotbeds have on the development of research competencies from their experience at the university. The research paradigm was framed in the quantitative - descriptive approach. A perception survey was applied to 82 students from five nurseries belonging to four research groups. The study manages to reveal that a large part of the students use digital technologies more frequently to search for relevant information for their research work and show a handling of specialized databases for this search.

KEYWORDS: research skills, higher education, research training.

\section{INTRODUCCIÓN}

La noción de competencia puede ser considerada como "atributos personales (capacidad, idoneidad), elementos de proceso (tener la habilidad, cierta destreza, ir al encuentro una cosa de otra, estar de acuerdo con, aspirar) y referencias a un producto (conseguir algo, aspirar a algo)" (López, 2016, p. 313); lo anterior de acuerdo a una perspectiva histórica, una etimológica y una semántica que, finalmente, abordarían lo que son habilidades cognitivas, capacidades, habilidades prácticas, destrezas, motivación, emociones y valores.

Siguiendo con el mismo autor, el concepto de competencia es algunas veces multiconceptual; incluye en su esencia el proceso de "articular, activar, integrar, sintetizar, movilizar y combinar los saberes (conocer, hacer y ser)" (p. 316). Así, según dicho autor, las competencias se refieren a una respuesta a las exigencias individuales o sociales y forman parte del ser integral que es la persona a través de los cambiantes y diversos contextos en que "participa, interactúa y aprende" (p. 316), logrando esto a cuenta de la ética, de una idoneidad, autonomía, flexibilidad y eficacia (López, 2016).
Acorde a lo anterior, Ribes (2011) sugiere que dentro de lo aprendido deben estar las normas, valores, diseños y pautas externas que influirán en la pertinencia de la actividad que se desempeñe; esto, a razón de que se es competente cuando "se aprenden desempeños y criterios de ejercicio del conocimiento" (p. 41). Por lo anterior, el presente autor asegura que el concepto de competencia deberá incluir en sí dos aspectos: "la especificación de actividades en relación con otras actividades, objetos y/o circunstancias, pero, además, debe incluir el criterio que dichas actividades y no otras satisfacen respecto del resultado, logro o efecto a tener en tal situación." (p.41). Además, se debe mencionar que las acepciones que agrega Granero (2005) dicho término ha ido avanzando en el tiempo de manera que "se adscribe a significados como "competir, rivalizar", entre otros. En el habla contemporánea toma las acepciones de autoridad, capacitación, cualificación, incumbencia y suficiencia" (p.11).

Es así que, desde los diferentes tipos de educación, el profesionalismo y la docencia, también se ha buscado esta competencia, capacidad y este poder desempeñar tareas particulares, ya que "El objetivo de la educación es hacer capaces a los estudiantes de desempeñar ciertas tareas vinculadas a una determinada ocupación o profesión" (Mulder, Weigel \& Collings, 2008, p.8), mismos autores que afirman que:

El concepto de competencia muestra interés, por tanto, en los objetivos significativos y en el contenido del aprendizaje que constituirá el desarrollo personal de los estudiantes y su posición en el dominio del conocimiento que mejor los prepare para funcionar de manera efectiva en la sociedad (p.3).

Ahora, de manera específica, las competencias investigativas dan cuenta de un proceso imbuido en el contexto capitalista, donde el fin buscado es el dinero, el material; teniendo en cuenta lo 
anterior, se dice que en la investigación "Se compite por fondos públicos y privados, por financiación para proyectos de investigación e incluso por consultorías con el afán de mejorar la situación financiera de instituciones y grupos de investigación." (Rivas, 2011, p. 35). Dicha competencia, depende del campo de ciencia con el que se trabaje para desarrollar rasgos de la personalidad, habilidades y conocimientos.

Es necesario entonces desglosar el concepto para entenderlo desde sus campos de participación; es por esto que se alude a Partingon (2002), citado en Rivas (2011), quien clasifica estas competencias en tres grupos; a saber: competencias sobre filosofía y epistemología, que tiene en cuenta los factores ética y filosofía dentro del diseño de la investigación y la esencia de esta; competencias sobre el proceso de la investigación, que incluye la "capacidad para teorizar y construir modelos, y las competencias de escritura científica, así como la habilidad relacional del investigador con los sujetos de investigación, la gestión de datos y ciertas características de personalidad del investigador"(p. 36) y, competencias sobre técnicas de investigación, que abordan los enfoques, métodos, técnicas y herramientas de investigación, mencionando además la investigación para la acción.

Así, es importante tener en cuenta algunas competencias en investigación, que como propone Cuevas, Guillén y Rocha (2011), pueden comportarse como un puente cognitivo para el aprendizaje significativo en la medida en que conducen de los saberes previos a saberes posteriores; como: la observación, la lectura, la expresión, la creatividad, el rigor, la socialización, la construcción, la estrategia, la problematización y la ética. Además, dichas competencias, pueden ayudar con la inclusión en los cambiantes sistemas (sociocultural, económico y político), promoviendo así el desarrollo individual y social, además de contribuir en la "formación de habilidades cognitivas de orden superior" (Campos, 2009, p. 1) que interesa a los estudiantes de educación superior, teniendo en cuenta la afirmación siguiente del mismo autor, a saber: "las capacidades investigativas deben ser inherentes a toda persona titulada como profesional." (p. 2); personas que deben tener en cuenta las "necesidades de carácter social, cultural, ambiental y político, que posee la sociedad actual y reconfigure su quehacer teórico-práctico en función de tales necesidades" (Campos, 2009, p. 4) para ser competentes en la producción y difusión del conocimiento.

Por otra parte, según Álvarez, Pérez y Suárez (2008), además de personas capaces de "desenvolverse en contextos cambiantes" ( $p$. 17), se demandan personas capaces de resolver problemas reflexiva y planificadamente; dicho autor resalta que el concepto de competencia dentro del campo de la educación se entiende desde un patrón constructivista y social a través del cual se plantea que el conocimiento es "fruto de la actividad del sujeto que tiene lugar en contextos sociales" (p. 20). Específicamente, dentro de la educación para la "realización personal, inclusión social, ciudadanía activa y empleabilidad en la sociedad del conocimiento" (Figel, 2009, párr. 3) se plantean a los ciudadanos desde el Ministerio de Educación Nacional, ocho competencias claves que entrelazan: "pensamiento crítico, creatividad, capacidad de iniciativa, resolución de problemas, evaluación del riesgo, toma de decisiones y gestión constructiva de los sentimientos" (párr. 5) y la comunicación en la lengua materna, la Comunicación en lenguas extranjeras, las Competencias matemática y las básicas en ciencia y tecnología, la competencia digital, aprender a aprender, las competencias sociales y cívicas, sentido de la iniciativa y espíritu de empresa y la conciencia y expresión culturales (Contreras RamírezParis, Ramírez-Paris Colmenares y Hernández Vergel, 2019). 
Se puede considerar que el enfoque de competencias nace en países como Inglaterra y Australia, quienes "[...]lo vieron como una herramienta útil para mejorar las condiciones de eficiencia, pertinencia y calidad de la capacitación laboral, y de este modo mejorar la productividad" (Larraín y González, 2007, p. 1), donde se promovían las competencias de: "gestión de recursos, relaciones interpersonales, gestión de información, comprensión sistémica y dominio tecnológico." (p. 2) para verse luego, según los autores, como enfoque clave en la formación de trabajadores productivos, para potenciar la calidad de los procesos de enseñanza en educación superior

En el ámbito internacional se han realizado investigaciones relevantes sobre las competencias investigativas en estudiantes universitarios; por ejemplo, Espinoza, Rivera y Tinoco (2016), apuntan la opción de "formar a los estudiantes en y para la investigación" ( $p$. construcción de un proyecto de investigación, es decir, que no se desarrollen las competencias investigativas en una única asignatura, sino a lo largo todo un proceso educativo y por medio del hecho de investigar con su sentido de búsqueda, el desarrollo de la curiosidad y el seguimiento de las fases investigativas. Los autores mencionan los motivos personales y sociales de incluir en los sistemas de educación la práctica investigativa: "en el plano personal, el hecho de investigar supone un ejercicio de madurez, responsabilidad y autogobierno que se muestra mediante un alto grado de autonomía de los sujetos." (p. 29) además, se puede deducir dentro de este plano el desarrollo laboral posterior a la educación superior; por su lado, dentro del ámbito social, la cultura investigativa. Esto implica

...conocimiento, manejo y participación en los proyectos sociales. Un saber que llevado a la práctica permite la integración y el intercambio social,

básicos para el desarrollo comunitario con base en la acción, el compromiso y la equidad, de ahí la importancia de procurar en los nuevos profesionales la aculturización investigativa (Espinoza, Rivera y Tinoco, 2016, p.p. 29 - 30).

Respecto al mismo tema Ángelo (2004) expone que el enfoque de competencias "coloca su articulación holística en los procesos constitutivos de la persona social, a la vez que le imprime, intencionalmente, una dirección transformadora definida a los procesos de aprendizaje social.", (p. 121) a través del mantenimiento de aprendizajes humanistas, crítico-reflexivos y autónomos-emancipatorios. A pesar de lo anterior, se ha visto que, a través de la historia, la investigación en Latinoamérica no se muestra alentadora en calidad, por lo que estas universidades tienen la necesidad de "utilizar de manera más eficiente sus recursos, preparar mejor para las profesiones de mercado, que son más competitivas que las tradicionales, fortalecer su capacidad de investigación y establecer puentes efectivos de colaboración y participación con la sociedad más amplia." (Schwartzman, 2013, párr. 8).

Siguiendo esta idea, según el precedente autor, después de reconocer, mediante conocimientos globales, profesionales y laborales, las necesidades y problemas de la realidad social, estos pasan a definirse por medio del diagnóstico, la práctica, la disciplina, el campo laboral y la meta de la institución; logrando así la comprensión y construcción de competencias profesionales específicas y abordando los desempeños laborales desde la educación universitaria, para la cual, según el mismo autor: [...] hay dos caminos posibles. Uno es organizar módulos y expresar directamente los conocimientos habilidades y actitudes en forma de comportamientos evaluables. La otra es un listado de los contenidos (tópicos o materias) que son necesarias y los objetivos 
de aprendizaje expresados en forma de comportamientos evaluables asociados a cada contenido, utilizando para estos fines el método tradicional de la planificación. Esta segunda manera, si bien es menos ortodoxa resulta más operativa para el trabajo con docentes universitarios. En ambos casos se pueden utilizar tablas de comportamientos observables que se encuentran disponibles en los manuales de pedagogía (Schwartzman, 2013).

También existen proyectos como Tuning Europa y América Latina (Bravo, 2007), que giran en torno al panorama de cambio social, de movilización estudiantil y de la respuesta que se les da a los empleadores respecto a títulos y capacitaciones en la práctica, que exige de las universidades a su vez una reforma; dicho proyecto se orienta hacia competencias genéricas y específicas a cada área temática de los graduados de primero y segundo ciclo. Aún más, el proyecto tiene un impacto directo en el reconocimiento académico, garantía y control de calidad, compatibilidad de los programas de estudio a nivel europeo, aprendizaje a distancia y aprendizaje permanente (p. 1).

Estas competencias, se abordan siempre desde los conocimientos y se organizaron por medio del proyecto en genéricas y específicas, como se citó antes: "si bien las competencias relacionadas con cada área de estudio son cruciales para cualquier título y se refieren a la especificidad propia de un campo de estudio las competencias genéricas identifican los elementos compartidos que pueden ser comunes a cualquier titulación, tales como la capacidad de aprender, de tomar decisiones, de diseñar proyectos, las destrezas administrativas, etc., que son comunes a todos o a la mayoría de las titulaciones (Bravo, 2007, p. 6).

Cabe mencionar además las 27 competencias que incluyen a toda profesión; aquellas instrumentales, interpersonales y sistémicas, según el proyecto:
1. Capacidad de abstracción, análisis y síntesis

2. Capacidad de aplicar los conocimientos en la práctica

3. Capacidad para organizar y planificar el tiempo

4. Conocimientos sobre el área de estudio y la profesión

5. Responsabilidad social y compromiso ciudadano

6. Capacidad de comunicación oral y escrita

7. Capacidad de comunicación en un segundo idioma

8. Comunicación

9. Capacidad de investigación

10. Capacidad de aprender y actualizarse permanentemente

11. Habilidades para buscar, procesar y analizar información procedente de fuentes diversas

12. Capacidad crítica y autocrítica

13. Capacidad para actuar en nuevas situaciones

14. Capacidad creativa

15. Capacidad para identificar, plantear y resolver problemas

16. Capacidad para tomar decisiones

17. Capacidad de trabajo en equipo

18. Habilidades interpersonales

19. Capacidad de motivar y conducir hacia metas comunes 
20. Compromiso con la preservación del medio ambiente

21. Compromiso con su medio sociocultural

22. Valoración y respeto por la diversidad y multiculturalidad

23. Habilidad para trabajar en contextos internacionales

24. Habilidad para trabajar en forma autónoma

25. Capacidad para formular y gestionar proyectos

26. Compromiso ético

27. Compromiso con la calidad (Bravo, 2007, p.p. 14-15).

En Colombia particularmente se han hecho trabajos importantes sobre este campo; Gutiérrez (2015) buscó "establecer un modelo

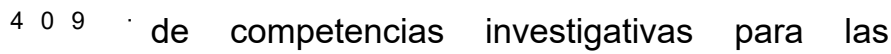
empresas (empresarios y profesionales) en el marco de la relación Universidad, Empresa y Estado (UEE) en Colombia." (p. 241); donde si bien las empresas han perdido la fe en la academia, necesitan articularse en pro de una producción, generando una reforma en las maneras de actuar en el contexto social, laboral e investigativo y donde el estado direccione los aportes e identifique sectores para contribuir juntos a la economía nacional colombiana.

También Reiban, De la Rosa y Zeballos (2017), realiza un acercamiento a las competencias investigativas de un profesor de educación superior y su evaluación, generando conclusiones en torno a dos alternativas de abordaje de las competencias investigativas: "a.) Partir de delimitar los aspectos organizativos, comunicacionales y colaborativos que debe evidenciar el profesor en las investigaciones a nivel universitario", y "b.) Partir de la misma lógica de la planeación y desarrollo de la investigación" (p. 402).

A razón de la calidad, del desarrollo social, económico e individual, se hace necesario abordar el tema de las competencias investigativas en la educación superior, trabajando con los y las estudiantes de los semilleros, extrayendo una reflexión en torno a las nuevas actuaciones que deben ser holísticas y tener en cuenta las transformaciones sociales y disciplinares en torno a la época actual.

\section{METODOLOGÍA}

Enfoque. El enfoque de la investigación es cuantitativo. La metodología utilizada para el desarrollo del estudio es de tipo descriptivo, debido a que se busca caracterizar las percepciones que poseen los estudiantes de un programa de educación superior, sobre el nivel de desarrollo de las competencias investigativas. Es decir, se pretende medir y recoger información de manera independiente sobre las dimensiones (Hernández, Fernández y Baptista, 2014) que conforman dicha competencia.

Diseño. El diseño es de tipo transversal descriptivo, estos "tienen como objetivo indagar la incidencia de las modalidades o niveles de una o más variables en una población" (Hernández et al, 2014, p. 155), recolectando información en un tiempo único.

Población y muestra. Los participantes del presente estudio fueron 82 estudiantes de cinco semilleros pertenecientes a cuatro grupos de investigación de la Universidad Francisco de Paula Santander (Cúcuta - Colombia).

Técnica e instrumentos. Para indagar sobre la percepción, se utilizó un cuestionario que consta de una serie de afirmaciones tipo escala de Likert, con cinco opciones de respuestas que van de 1 (Nunca) a 5 (Siempre). El baremo para el instrumento utilizado se muestra en la tabla 1. 
Tabla 1. Baremo del instrumento.

\begin{tabular}{|c|c|c|}
\hline Rango de actuación & Categorías & Criterios \\
\hline $1-1,99$ & Nunca & Muy baja \\
\hline $2-2,99$ & Pocas veces & Baja \\
\hline $3-3,99$ & Eventualmente & Moderada \\
\hline $4-4,99$ & Casi siempre & Alta \\
\hline 5 & Siempre & Muy alta \\
\hline
\end{tabular}

El instrumento utilizado consta de 36 ítems, relacionados con cuatro dominios: Búsqueda de información; técnico; metodológico y comunicación de resultados. Dichos dominios, se relacionan de manera directa con lo expresado por Hernández-Suárez, PradaNúñez, \& Avendaño-Castro, 2020) y por Hernández-Suárez, Prada-Núñez, \& GamboaSuarez, 2021).

Ahora bien, con el fin de validar el contenido, se envió el cuestionario inicial a tres expertos, quienes dieron recomendaciones de forma sobre cada ítem evaluado. Además, la confiabilidad, evaluada por el alfa de Cronbach, obtuvo valor aceptable de 0.88 . El proceso de análisis de los datos se realizó utilizando el SPSS v25.

\section{RESULTADOS}

Los resultados se presentan para cada uno de los dominós evaluados, y se examinan desde las percepciones de los estudiantes, en atención a las frecuencias y porcentajes obtenidos.

Tabla 1. Dominio en la búsqueda de información

\begin{tabular}{|c|c|c|c|c|}
\hline & Ítem & Opciones & No & Porcentaje \\
\hline \multirow{15}{*}{ 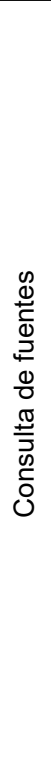 } & \multirow{5}{*}{$\begin{array}{l}\text { 1. Busca información relevante en libros y revistas } \\
\text { académicas en la biblioteca. }\end{array}$} & Nunca & 4 & $2,94 \%$ \\
\hline & & Pocas veces & 64 & $47,06 \%$ \\
\hline & & Eventualmente & 28 & $20,59 \%$ \\
\hline & & Casi siempre & 32 & $23,53 \%$ \\
\hline & & Siempre & 8 & $5,88 \%$ \\
\hline & \multirow{5}{*}{ 2. Busca información relevante en revistas electrónicas. } & Nunca & 8 & $5,88 \%$ \\
\hline & & Pocas veces & 12 & $8,82 \%$ \\
\hline & & Eventualmente & 48 & $35,29 \%$ \\
\hline & & Casi siempre & 36 & $26,47 \%$ \\
\hline & & Siempre & 32 & $23,53 \%$ \\
\hline & \multirow{5}{*}{$\begin{array}{l}\text { 3. Busca información en } \\
\text { bases de datos. }\end{array}$} & Nunca & 4 & $2,94 \%$ \\
\hline & & Pocas veces & 36 & $26,47 \%$ \\
\hline & & Eventualmente & 20 & $14,71 \%$ \\
\hline & & Casi siempre & 60 & $44,12 \%$ \\
\hline & & Siempre & 16 & $11,76 \%$ \\
\hline
\end{tabular}




\begin{tabular}{|c|c|c|c|c|}
\hline \multirow{15}{*}{ 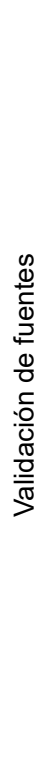 } & \multirow{5}{*}{$\begin{array}{l}\text { 4. Elabora fichas de registro de información relevante } \\
\text { asociada con sus antecedentes. }\end{array}$} & Nunca & 24 & $17,65 \%$ \\
\hline & & Pocas veces & 48 & $35,29 \%$ \\
\hline & & Eventualmente & 48 & $35,29 \%$ \\
\hline & & Casi siempre & 12 & $8,82 \%$ \\
\hline & & Siempre & 4 & $2,94 \%$ \\
\hline & \multirow{5}{*}{$\begin{array}{l}\text { 5. Emplea un sistema de referencias para dar crédito a } \\
\text { las fuentes consultadas. }\end{array}$} & Nunca & 20 & $14,71 \%$ \\
\hline & & Pocas veces & 16 & $11,76 \%$ \\
\hline & & Eventualmente & 28 & $20,59 \%$ \\
\hline & & Casi siempre & 44 & $32,35 \%$ \\
\hline & & Siempre & 28 & $20,59 \%$ \\
\hline & & Nunca & 16 & $11,76 \%$ \\
\hline & & Pocas veces & 24 & $17,65 \%$ \\
\hline & b. Distingue evidenclas clentiricas de otro tipo de & Eventualmente & 32 & $23,53 \%$ \\
\hline & & Casi siempre & 60 & $44,12 \%$ \\
\hline & & Siempre & 4 & $2,94 \%$ \\
\hline \multirow{10}{*}{ 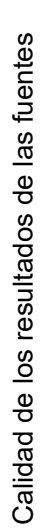 } & \multirow{5}{*}{$\begin{array}{l}\text { 7. Contrasta planteamiento y postura de } \\
\text { diferentes autores acerca del tema en estudio. }\end{array}$} & Nunca & 8 & $5,88 \%$ \\
\hline & & Pocas veces & 20 & $14,71 \%$ \\
\hline & & Eventualmente & 52 & $38,24 \%$ \\
\hline & & Casi siempre & 20 & $14,71 \%$ \\
\hline & & Siempre & 36 & $26,47 \%$ \\
\hline & \multirow{5}{*}{$\begin{array}{l}\text { 8. Realiza evaluación crítica de las diferentes } \\
\text { posturas teóricas revisadas. }\end{array}$} & Nunca & 8 & $5,88 \%$ \\
\hline & & Pocas veces & 36 & $26,47 \%$ \\
\hline & & Eventualmente & 48 & $35,29 \%$ \\
\hline & & Casi siempre & 20 & $14,71 \%$ \\
\hline & & Siempre & 24 & $17,65 \%$ \\
\hline
\end{tabular}

La tabla 1, muestra los ítems del 1 al 8 , los cuales hacen referencia al dominio en la búsqueda de información con respecto a sus indicadores consulta de fuentes, su validación y calidad de los resultados de dichas fuentes. Estos resultados revelaron que en el ítem 1, el $47,06 \%$ de los encuestados, pocas veces buscan información relevante en libros y revistas académicas en la biblioteca. Mientras que un $23,53 \%$ casi siempre lo hace y otros eventualmente lo hacen $(20,59 \%)$. El resto siempre $(5,88 \%)$ y nuca $(2,94 \%)$ investigan la información en la biblioteca. En el caso del ítem 2, el 50,00\% opinan que siempre y casi siempre indagan información relevante en revistas electrónicas. El resto, señaló que eventualmente investiga en revistas electrónicas $(35,29 \%)$ y el $14,70 \%$ nunca o pocas veces lo hacen.
Con relación al ítem 3, el 55,88\% declararon que esas informaciones indagadas siempre y casi siempre provienen de bases de datos. La del resto, vienen eventualmente $(14,71 \%)$ y pocas veces $(26,47 \%)$ y solo un $2,94 \%$ nunca.

Por otro lado, al examinar el ítem 4, se constató que el $52,94 \%$ de los encuestados nunca y pocas veces elabora fichas de registro de información relevante asociada con sus antecedentes. Mientras que un $35,29 \%$ mencionó que eventualmente registra dicha información investigada. El resto, 11.76\% siempre y casi siempre lo elaboran. En cuanto al ítem 5 , se verificó que el $52,94 \%$ de los estudiantes encuestados consideran siempre y casi siempre emplear un sistema de referencias para dar crédito a las fuentes consultadas. 
Otros discentes eventualmente dan el crédito a la consulta realizada $(20,59 \%)$. El resto, nuca o casi nuca $(26,47 \%)$.

En referencia al ítem 6, se confirmó que el $47,06 \%$ de los encuestados distinguen siempre y casi siempre las evidencias científicas de otro tipo de evidencias. Mientras que un 23,53\% eventualmente las diferencia. El resto nuca y casi nunca $(29,41 \%)$.

En este sentido, se determinó a través del ítem 7 , que el $41,18 \%$ de los encuestados contrastan siempre y casi siempre los planteamientos y posturas de diferentes autores acerca del tema en estudio. Otros eventualmente lo contrastan $(38,24 \%)$ y el resto nunca y casi nunca ((20,59\%). En lo relativo al ítem 8, se comprobó que el $32,36 \%$ de los encuestados siempre y casi siempre realizan evaluaciones críticas de las diferentes posturas teóricas revisadas. EI resto, lo efectúan eventualmente $(35,29 \%)$, pocas veces $(26,47 \%)$ y nunca $(5,88 \%)$.

Atendiendo estos primeros resultados, se evidencia que lo aprendido en la consulta de fuentes, así como su validación y evaluación influirá en la eficacia de las actividades realizadas, y por ende en la práctica de la búsqueda de información y hacia un dominio de competencias investigas la cuales actúan en el discernimiento de la actuación del individuo, como lo plantea Ribes (2011) se es competente cuando se ejercitan el trabajo realizado y criterios de ejercicio del conocimiento dado que asegura la especificación de la tarea que realiza y los resultados logrados en tal situación.

Todo lo anteriormente expuesto, permite inferir que se ha buscado que los estudiantes forjen esta competencia en el dominio de las consultas de las fuentes de información, dado que son tareas vinculadas a la formación y determinada ocupación (Mulder, Weigel \& Collings, 2008). En ese contexto, los resultados coinciden por el argumento que presentan estos autores precitados, que con el desarrollo personal de los estudiantes y su posición en el dominio del conocimiento buscan, validan y evaluar las fuentes que permitan lograr los objetivos. Lo que conduce a realizar un análisis de la dimensión: Dominio en la búsqueda con respecto a sus indicadores para categorizar las competencias en el proceso investigativos, de allí que se presenta la siguiente tabla 2 .

Tabla 2. Promedio de medias de la dimensión: Dominio en la búsqueda de información

\begin{tabular}{|llll|}
\hline Indicadores & Ítems & Promedio & Categoría \\
\hline Consulta de fuentes & 1 al 3 & 3,24 & Moderada \\
\hline Validación de fuentes & 4 al 6 & 2,95 & Baja \\
\hline $\begin{array}{l}\text { Calidad de los } \\
\text { resultados de las } \\
\text { fuentes }\end{array}$ & 7 y 8 & 3,26 & Moderada \\
\hline Promedio General & & $\mathbf{3 , 1 5}$ & Moderada \\
\hline
\end{tabular}


En los resultados obtenidos, se puede visualizar que los mayores puntajes lo alcanzaron los indicadores consultas de fuentes y la calidad de los resultados de las fuentes con un promedio de media de 3,24 y 3,26 respectivamente; ubicándose en la categoría nivel moderado, el indicador validación de las fuentes obtuvo el menor promedio con un valor de 2,95 y una categoría de nivel bajo. El promedio general para la dimensión fue de 3,15 que la coloca, de igual manera, en la categoría de nivel moderado. Por tanto, los resultados evidencian que los estudiantes en formación consultan fuentes y obtienen en resultados de calidad, pero algunas veces sin validar dichas fuentes con lo que podría dar indicios para elaborar información en el análisis y reflexión de los elementos identificados previamente en las tablas de frecuencias estadísticas con el fin de orientar el dominio de la pesquisa con el arqueo de teoría posturas y opiniones de los autores consultados.

Tabla 3 Dominio técnico-Uso de software

\begin{tabular}{|c|c|c|c|c|}
\hline & Ítem & Opciones & No & Porcentaje \\
\hline & \multirow{5}{*}{ 9.Word } & Nunca & 0 & $0,00 \%$ \\
\hline & & Pocas veces & 0 & $0,00 \%$ \\
\hline & & Eventualmente & 16 & $11,76 \%$ \\
\hline & & Casi siempre & 60 & $44,12 \%$ \\
\hline & & Siempre & 60 & $44,12 \%$ \\
\hline & \multirow{5}{*}{ 10. Excel } & Nunca & 8 & $5,88 \%$ \\
\hline & & Pocas veces & 28 & $20,59 \%$ \\
\hline & & Eventualmente & 36 & $26,47 \%$ \\
\hline & & Casi siempre & 44 & $32,35 \%$ \\
\hline & & Siempre & 20 & $14,71 \%$ \\
\hline & \multirow{5}{*}{ 11. Power Point } & Nunca & 0 & $0,00 \%$ \\
\hline & & Pocas veces & 0 & $0,00 \%$ \\
\hline & & Eventualmente & 8 & $5,88 \%$ \\
\hline & & Casi siempre & 60 & $44,12 \%$ \\
\hline & & Siempre & 68 & $50,00 \%$ \\
\hline \multirow{5}{*}{ 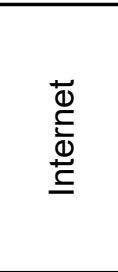 } & \multirow{5}{*}{ 12. Internet } & Nunca & 0 & $0,00 \%$ \\
\hline & & Pocas veces & 0 & $0,00 \%$ \\
\hline & & Eventualmente & 8 & $5,88 \%$ \\
\hline & & Casi siempre & 52 & $38,24 \%$ \\
\hline & & Siempre & 76 & $55,88 \%$ \\
\hline \multirow{5}{*}{ 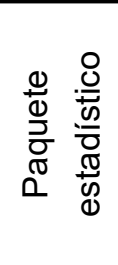 } & \multirow{5}{*}{$\begin{array}{l}\text { 13. Paquetes } \\
\text { estadísticos }\end{array}$} & Nunca & 12 & $8,82 \%$ \\
\hline & & Pocas veces & 16 & $11,76 \%$ \\
\hline & & Eventualmente & 60 & $44,12 \%$ \\
\hline & & Casi siempre & 32 & $23,53 \%$ \\
\hline & & Siempre & 16 & $11,76 \%$ \\
\hline
\end{tabular}




\begin{tabular}{|c|c|c|c|c|c|}
\hline \multirow{10}{*}{ 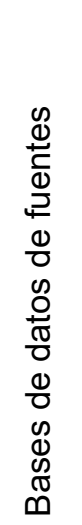 } & \multirow{6}{*}{ 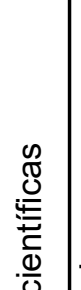 } & \multirow{5}{*}{$\begin{array}{l}\text { 14. Base de } \\
\text { datos general } \\
\text { (Google } \\
\text { Scholar) }\end{array}$} & Nunca & 20 & $14,71 \%$ \\
\hline & & & Pocas veces & 16 & $11,76 \%$ \\
\hline & & & Eventualmente & 44 & $32,35 \%$ \\
\hline & & & Casi siempre & 32 & $23,53 \%$ \\
\hline & & & Siempre & 24 & $17,65 \%$ \\
\hline & & & Nunca & 24 & $17,65 \%$ \\
\hline & $\overline{0}$ & 15. Base & Pocas veces & 20 & $14,71 \%$ \\
\hline & $\frac{\mathscr{O}}{E}$ & de datos & Eventualmente & 52 & $38,24 \%$ \\
\hline & $\frac{\bar{\Phi}}{\bar{\sigma}}$ & de fuentes & Casi siempre & 16 & $11,76 \%$ \\
\hline & $\ddot{\pi}$ & & Siempre & 24 & $17,65 \%$ \\
\hline
\end{tabular}

En la tabla 2, se referencia lo relacionado con el dominio técnico sobre paquete ofimático, Internet, paquete estadístico, bases de datos de fuentes académicas y científicas. Donde se muestra que el ítem 9, que el $88,24 \%$ de los encuestados dominan el procesador de texto Word. El resto lo usa eventualmente $(11,76 \%)$. Mientras que en el ítem 10, se evidenció que el $47,06 \%$ domina la hoja de cálculo Excel. Otros, lo dominan eventualmente en algunas funciones $(26,47 \%)$ y pocas veces y nuca lo dominan $(26,47 \%)$. Entretanto que en el ítem 11 , se comprobó que $94,12 \%$ domina la herramienta PowerPoint.

Con respecto al ítem 12, se probó que $94,12 \%$ de los encuestados dominan la internet. A propósito del ítem 13, se conoció que el $35,29 \%$ siempre y casi siempre dominan los paquetes estadísticos. Otros, eventualmente $(44,12 \%)$ y pocas veces y nunca $(20,58 \%)$

Con respecto al ítem 14, se demostró que $41,15 \%$ de los encuestados siempre y casi siempre dominan Base de datos general. El resto eventualmente $(32,35 \%)$ y pocas veces y nunca $(2647 \%)$. En lo referente al ítem 15 , se pudo evidenciar que el $38,24 \%$ dominan eventualmente las bases de datos de fuentes especializadas. Otro $32,36 \%$ pocas veces y nunca. Mientras que el $29,41 \%$ siempre y casi siempre dominan dichas bases de datos de especializadas.
Estos resultados de los ítems del 9 al 15, demuestran dominio técnico de los encuestados en cuanto al procesador texto (Word) y las herramientas de presentación PowerPoint, aunque no todos presentan un dominio de las herramientas de la hoja de cálculo (Excel) y paquetes estadísticos, los cuales son fundamentales para los análisis estadísticos descriptivos e inferenciales de los procesos investigativos. De igual modo, se encuentra una diferencia significativa en el dominio de internet para buscar fuentes de información y las bases de datos generales y especializadas consultadas, pues un tercio de los encuestados poco prestan atención a dichas fuentes expertas o académicas en los temas estudiados. No obstante, se evidencian algunas competencias en investigación, las cuales podría ser un puente cognitivo dicho aprendizaje (Cuevas, Guillén y Rocha (2011).

Todo lo anterior trae como consecuencia una comparación de la dimensión: Dominio técnico referente a sus indicadores para realizar la categorización respectiva en la misma, de allí que se muestra la tabla 4. 
Tabla 4. Promedio de medias de la dimensión Dominio técnico-uso de software

\begin{tabular}{|llll|}
\hline Indicadores & Ítems & Promedio & Categoría \\
\hline Paquete ofimático & 9 al 11 & 4,02 & Alta \\
\hline Internet & 12 & 4,50 & Alta \\
\hline Paquete estadístico & 13 & 3,18 & Moderada \\
\hline Bases de datos & 14 y 15 & 3,07 & Moderada \\
\hline Promedio General & & $\mathbf{3 , 6 9}$ & Moderada \\
\hline
\end{tabular}

Según los resultados obtenidos para la Dimensión Dominio técnico-uso de software, en el presente gráfico, se puede observar que los indicadores paquete ofimático e internet obtuvieron unos promedios en las medias de 4,02 y 4,50 respectivamente; asentándose cada uno en la categoría nivel Alta. En cuanto al indicador paquete estadístico, alcanzó un promedio en sus medias de 3,18 y una categoría de nivel Mejorado y el indicador bases de datos una categoría de nivel mejorado con
3,07 puntos en su valor de las medias. Siendo el promedio general para la dimensión de 3,69 que la ubicó, de igual manera, en la categoría de nivel Mejorado.

Por consiguiente, los resultados demuestran que los estudiantes, llevan los saberes técnicos de usos de software previos a saberes especializados posteriores; que pueden fomentar aún más las habilidades instrumentales y promover el desarrollo individual en el proceso de investigación.

Tabla 5. Dominio metodológico

\begin{tabular}{|c|c|c|c|c|}
\hline & Ítem & Opciones & No & Porcentaje \\
\hline \multirow{10}{*}{ 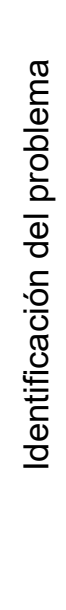 } & \multirow[t]{5}{*}{$\begin{array}{l}\text { 16. Plantea el problema a resolver a través de } \\
\text { la investigación. }\end{array}$} & Nunca & 8 & $5,88 \%$ \\
\hline & & Pocas veces & 0 & $0,00 \%$ \\
\hline & & Eventualmente & 28 & $20,59 \%$ \\
\hline & & Casi siempre & 76 & $55,88 \%$ \\
\hline & & Siempre & 24 & $17,65 \%$ \\
\hline & \multirow{5}{*}{$\begin{array}{l}\text { 17. Define una pregunta de investigación que } \\
\text { ayuda a resolver el problema planteado. }\end{array}$} & Nunca & 4 & $2,94 \%$ \\
\hline & & Pocas veces & 4 & $2,94 \%$ \\
\hline & & Eventualmente & 24 & $17,65 \%$ \\
\hline & & Casi siempre & 80 & $58,82 \%$ \\
\hline & & Siempre & 24 & $17,65 \%$ \\
\hline
\end{tabular}




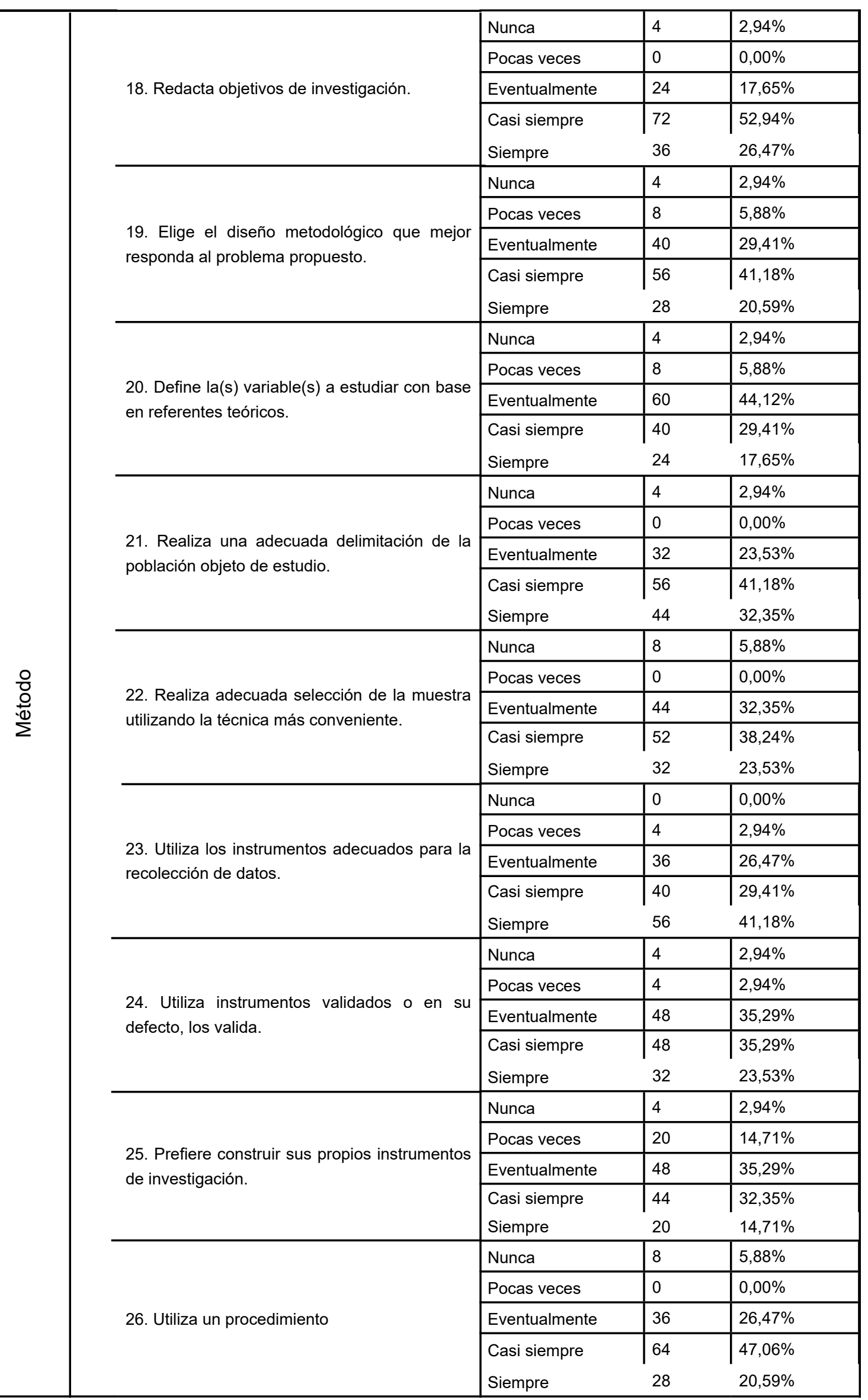


Los datos registrados en la tabla 5. Demostraron que para el ítem 16 , el $73,53 \%$ de los encuestados dijeron que siempre y casi siempre Plantea el problema a resolver a través de la investigación. Otro $20,59 \%$ lo hace eventualmente. En lo que se refiere al ítem 17, cabe destacar que el $76,47 \%$ estimó que siempre y casi siempre definen una pregunta de investigación que ayude a resolver la problemática planteada. Mientras que otro $17,65 \%$ señalo que lo precisan eventualmente. En cuanto al ítem 18, el 78,41\% de los estudiantes encuestados manifestaron que siempre y casi siempre redactan objetivos de investigación. Mientras que en el ítem 19, el $61.77 \%$ declararon que eligen el diseño metodológico que mejor responda al problema propuesto. Pero lo encontrado en el ítem 20, demuestra que el $47,06 \%$ de los encuestados siempre y casi siempre definen las variables a estudiar con base a los referentes teóricos. Mientras que un $44,12 \%$ eventualmente las define.

Igualmente se halló en el ítem 21, que el 73,53\% siempre y casi siempre realizan una adecuada delimitación de la población objeto de estudio y otro grupo representado por el $23,53 \%$ lo hacen eventualmente.

Los datos obtenidos en el ítem 22, reflejan que el $61,77 \%$ de estos encuestados siempre y casi siempre realizan adecuada selección de la muestra utilizando la técnica más conveniente, otro grupo lo hace eventualmente $(32,35 \%)$. Por otro lado, en relación al ítem 23 , El 70,53\% manifestaron que utilizan los instrumentos adecuados para la recolección de datos. Mientras que el $26,47 \%$ los utilizas eventualmente. En lo relativo al ítem 24 , el $58,82 \%$ siempre y casi siempre utiliza instrumentos validados o en su defecto, los valida. Entre tanto, que el 35,29\% los usa eventualmente. Por ello, en el ítem 25 , los encuestados señalan que siempre y casi siempre prefieren construir sus propios instrumentos de investigación $(47,06 \%)$ y otro $35,29 \%$ los construye eventualmente. En lo concerniente al ítem 26, estos encuestados manifestaron en un $67,65 \%$ que utilizan un procedimiento metodológico y otro $26,74 \%$ eventualmente.

Estos resultados indican, que el mayor porcentaje de respuestas entre los ítems 16,17, 18, 19, 20. $21,22,23,24,15$ y 26 , emplean los procesos inherentes con el trabajo de investigación. Lo cual está en concordancia con los datos encontrados en las dimensiones anteriores. Bajo esta perspectiva, estos resultados coinciden con las afirmaciones de Espinoza, Rivera y Tinoco (2016); Gamboa, Vargas y Hernández (2017), Villamizar-Acevedo, Lozano-León \& SierraGaravito (2017) y Gamboa (2016), quienes señalan que la opción de formar a los estudiantes en y para la investigación permite el desarrollo de las competencias investigativas no en una única asignatura, sino a lo largo del proceso formativo en toda la dimensión curricular del programa académico, por ello, resulta necesario presentar los promedios de medias con el fin de categorizar la dimensión: Habilidades Gerenciales en el Empleo de la Web 2.0 con relación a sus indicadores (ver tabla 6 ) 
Tabla 6. Promedio de medias de la dimensión: Dominio metodológico

\begin{tabular}{|llll|}
\hline Indicadores & \multicolumn{1}{l}{ Ítems } & Promedio & Categoría \\
\hline $\begin{array}{l}\text { Identificación } \\
\text { problema }\end{array}$ & del & & \\
& 16 al 18 & 3,88 & Moderada \\
\hline Método & 19 al 26 & 3,75 & Moderada \\
\hline Promedio General & & $\mathbf{3 , 8 2}$ & Moderada \\
\hline
\end{tabular}

Dentro de la Dimensión: Dominio metodológico, se distinguió que el indicador: identificación del problema, alcanzó un promedio de media de 3,88 ubicándose en la categoría nivel moderado. Mientras que el indicador método, logró un promedio de medias de 3,75 situándose en una categoría de nivel moderado. A causa de estos, el promedio general para la dimensión fue de 3,82 , se situó en la categoría de nivel moderado.
En consecuencia, se evidencia que los estudiantes participantes de esta investigación podrían presentar progresos significativos al querer desempeñar sus procesos investigativos al emplear un dominio metodológico, y por medio del hecho de investigar con su sentido de búsqueda, el desarrollo de la indagación y la persecución de las fases investigativas.

Tabla 7. Dominio - Comunicación de los resultados

\begin{tabular}{|c|c|c|c|c|}
\hline \multicolumn{2}{|c|}{ Ítem } & Opciones & No & Porcentaje \\
\hline \multirow{20}{*}{ 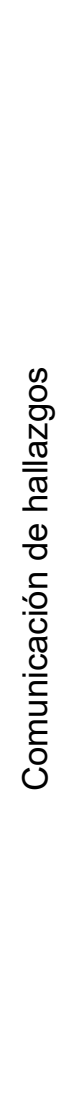 } & \multirow{5}{*}{ 27. Describe en texto la información obtenida. } & Nunca & 0 & $0,00 \%$ \\
\hline & & Pocas veces & 16 & $11,76 \%$ \\
\hline & & Eventualmente & 28 & $20,59 \%$ \\
\hline & & Casi siempre & 68 & $50,00 \%$ \\
\hline & & Siempre & 24 & $17,65 \%$ \\
\hline & \multirow{5}{*}{$\begin{array}{l}\text { 28. Recurre al uso de tablas o gráficas para } \\
\text { comunicar la información obtenida. }\end{array}$} & Nunca & 8 & $5,88 \%$ \\
\hline & & Pocas veces & 28 & $20,59 \%$ \\
\hline & & Eventualmente & 28 & $20,59 \%$ \\
\hline & & Casi siempre & 60 & $44,12 \%$ \\
\hline & & Siempre & 12 & $8,82 \%$ \\
\hline & \multirow{5}{*}{$\begin{array}{l}\text { 29. Presenta conclusiones congruentes con } \\
\text { la pregunta de investigación. }\end{array}$} & Nunca & 4 & $2,94 \%$ \\
\hline & & Pocas veces & 8 & $5,88 \%$ \\
\hline & & Eventualmente & 40 & $29,41 \%$ \\
\hline & & Casi siempre & 60 & $44,12 \%$ \\
\hline & & Siempre & 24 & $17,65 \%$ \\
\hline & \multirow{5}{*}{$\begin{array}{l}\text { 30. Redacta el informe de investigación con } \\
\text { orden evidenciando la estructura } \\
\text { metodológica. }\end{array}$} & Nunca & 4 & $2,94 \%$ \\
\hline & & Pocas veces & 12 & $8,82 \%$ \\
\hline & & Eventualmente & 60 & $44,12 \%$ \\
\hline & & Casi siempre & 32 & $23,53 \%$ \\
\hline & & Siempre & 28 & $20,59 \%$ \\
\hline
\end{tabular}




\begin{tabular}{|c|c|c|c|}
\hline \multirow{5}{*}{$\begin{array}{l}\text { 31. Escribe el informe de investigación con } \\
\text { claridad en la redacción. }\end{array}$} & Nunca & 0 & $0,00 \%$ \\
\hline & Pocas veces & 16 & $11,76 \%$ \\
\hline & Eventualmente & 36 & $26,47 \%$ \\
\hline & Casi siempre & 64 & $47,06 \%$ \\
\hline & Siempre & 20 & $14,71 \%$ \\
\hline \multirow{5}{*}{$\begin{array}{l}\text { 32. Aplica las normas de referenciación } \\
\text { adecuadas. }\end{array}$} & Nunca & 4 & $2,94 \%$ \\
\hline & Pocas veces & 8 & $5,88 \%$ \\
\hline & Eventualmente & 24 & $17,65 \%$ \\
\hline & Casi siempre & 88 & $64,71 \%$ \\
\hline & Siempre & 12 & $8,82 \%$ \\
\hline \multirow{5}{*}{$\begin{array}{l}\text { 33. Presenta en anexos la información } \\
\text { necesaria para complementar lo descrito en el } \\
\text { informe de investigación. }\end{array}$} & Nunca & 4 & $2,94 \%$ \\
\hline & Pocas veces & 12 & $8,82 \%$ \\
\hline & Eventualmente & 40 & $29,41 \%$ \\
\hline & Casi siempre & 64 & $47,06 \%$ \\
\hline & Siempre & 16 & $11,76 \%$ \\
\hline \multirow{3}{*}{$\begin{array}{l}\text { 34. Prepara un informe de investigación para } \\
\text { su publicación. }\end{array}$} & Nunca & 12 & $8,82 \%$ \\
\hline & Pocas veces & 24 & $17,65 \%$ \\
\hline & Eventualmente & 32 & $23,53 \%$ \\
\hline & Casi siempre & 56 & $41,18 \%$ \\
\hline & Siempre & 12 & $8,82 \%$ \\
\hline \multirow{5}{*}{$\begin{array}{l}\text { 35. Redacta un artículo de un informe de } \\
\text { investigación para su posterior publicación. }\end{array}$} & Nunca & 24 & $17,65 \%$ \\
\hline & Pocas veces & 32 & $23,53 \%$ \\
\hline & Eventualmente & 40 & $29,41 \%$ \\
\hline & Casi siempre & 32 & $23,53 \%$ \\
\hline & Siempre & 8 & $5,88 \%$ \\
\hline \multirow{5}{*}{$\begin{array}{l}\text { 36. Presenta en eventos académicos los } \\
\text { resultados de su investigación. }\end{array}$} & Nunca & 24 & $17,65 \%$ \\
\hline & Pocas veces & 32 & $23,53 \%$ \\
\hline & Eventualmente & 36 & $26,47 \%$ \\
\hline & Casi siempre & 28 & $20,59 \%$ \\
\hline & Siempre & 16 & $11,76 \%$ \\
\hline
\end{tabular}

La presente tabla 7 muestra los ítems del 27 al 36, los cuales hacen referencia al dominio de comunicación de resultados. Estos revelaron que en el ítem 27 , el $67,65 \%$ de los participantes encuestados, manifestaron que siempre y casi siempre describen en texto la información obtenida. Mientras, que el 20,59\% eventualmente lo hace. En el caso del ítem 28 , el $52,94 \%$ siempre y casi siempre recurren al uso de tablas o gráficas para comunicar la información obtenida. Otros dijeron que eventualmente $(20,59 \%)$ y pocas veces $(20,59)$ acuden al uso de tablas o gráficos.

Por otra parte, referente al ítem 29, se evidenció que el $61,77 \%$ siempre y casi siempre presenta conclusiones congruentes con la pregunta de investigación, mientras que el $29,41 \%$ eventualmente muestran los colofones $y$ terminaciones de los trabajos 
de indagación. En relación con el ítem 30, el $44,12 \%$ declararon que siempre y casi siempre redactan el informe de investigación con orden evidenciando la estructura metodológica. Otro $44,12 \%$ eventualmente transcriben el informe de indagación donde se evidencia lo metodológico. Por otro lado, al examinar el ítem 31, se constató que el $61,77 \%$ de los encuestados siempre y casi siempre escriben el informe de investigación con claridad en la redacción, mientras que otro grupo dice lo hace eventualmente $(26,47 \%)$.

En correspondencia al ítem 32 , el $73,53 \%$ de los encuestados revelaron que siempre y casi siempre aplican las normas de referenciación adecuadas. Entretanto que existen encuestados que aplican las normas eventualmente $(17,65 \%)$. Lo relativo al ítem 33 , se encontró que el $58,82 \%$ presenta en anexos la información necesaria para complementar lo descrito en el informe de investigación entre las alternativas siempre y casi siempre y un $29,41 \%$ en la opción eventualmente. En cuanto al ítem 34, se conoció que el $50,00 \%$ siempre y casi siempre presentan un informe de investigación para su publicación. El resto, eventualmente $(23,53 \%)$ y pocas veces $(17,65 \%)$. De esa manera, se determinó en el ítem 35 , que el $29,41 \%$ de los encuestados siempre y casi siempre redactan un artículo de un informe de investigación para su posterior publicación, otros lo hacen eventualmente $(29,41 \%)$ y pocas veces el $23,53 \%$ y nunca un $17,65 \%$. De allí, que en el ítem 36 , se evidenciara que el $32,35 \%$ podría siempre y casi siempre presentar en eventos académicos los resultados de sus investigaciones. El resto, eventualmente $(26,47 \%)$, pocas veces $(23,53 \%)$ y nunca el $17,65 \%$.

De los resultados obtenidos, se desprende que hay un acercamiento a las competencias investigativas, pues se evidencia la delimitación de aspectos organizativos, estructurales y comunicativos de la misma lógica y desarrollo de la indagación (Reiban, de la Rosa y Zeballos, 2017; Urbina, Gamboa y Prada, 2020). De ahí, que se formen competencias investigativas, las cuales puedan servir a los futuros profesionales, como lo plantea Gutiérrez (2015) al establecer un modelo de competencias investigativas para las empresas (empresarios y profesionales).

Adicionalmente, se hace necesario dar a conocer la siguiente tabla sobre los promedios de medias con el fin de categorizar la dimensión: Dominio-Comunicación de los resultados con relación a sus indicadores (ver tabla 8 ).

Tabla 8. Promedio de medias de la dimensión: Comunicación de resultados

: Comunicación de los resultados

\begin{tabular}{|llll|}
\hline Indicadores & \multicolumn{1}{l}{ Ítems } & Promedio & Categoría \\
\hline $\begin{array}{l}\text { Comunicación } \\
\text { Hallazgos }\end{array}$ & 27 al 34 & 3,54 & Moderada \\
\hline Entrega de informe & 35 y 36 & 2,81 & Moderada \\
\hline Promedio General & & $\mathbf{3 , 1 8}$ & Moderada \\
\hline
\end{tabular}


Según los resultados de los instrumentos aplicados, se observó que el indicador comunicación de hallazgos se encuentran en una categoría de nivel moderado al obtener un valor en el promedio de media de 3,54 alcanzando el mayor puntaje de la Dimensión: Comunicación de los resultados. Mientras que el indicador difusión de resultados de investigación en eventos o publicaciones promedió la menor puntuación al lograr 2,81 entre las medias para ubicarse en una categoría de nivel baja. El promedio general para la dimensión fue de 3,18 puntos que lo sitúa en una categoría de nivel moderado. Por lo tanto, los resultados obtenidos demandan continuar con la articulación de competencias investigativas de los discentes en formación, con el fin de conseguir los conocimientos necesarios y adquirir habilidades, destrezas, procedimientos en los procesos de investigación.

\section{CONCLUSIONES}

$\begin{array}{lll}4 & 21 & 1\end{array}$ El estudio logra revelar que gran parte de los estudiantes utilizan con mayor frecuencia las tecnologías digitales para la búsqueda de información relevante para sus trabajos de investigación y muestran un manejo de las bases de datos especializadas para esta búsqueda. En contraste este estudio también revela que son escasos los estudiantes que se dirigen a la biblioteca a consultar libros y revistas impresas.

La investigación demostró que gran parte de los estudiantes encuestados señalan un buen dominio de los medios ofimáticos básicos, sin embargo reconocen que no tienen un dominio de software de investigación para Análisi de datos cualitativos o cuantitativos.

Otro aspecto importante que se logró evidenciar en el estudio, es la capacidad de contrastación teórica que tiene los estudiantes que participaron en la investigación, puesto que, según los datos recolectados estos triangulan los planteamientos y posturas de diferentes autores con tema en estudio.

Un elemento relevante que es necesario mencionar, es el reconocimiento por parte de los estudiantes del buen manejo de la redacción científica y las normas de citas y referenciación; no solo en sus propuestas de investigación sino en los trabajos de otras asignaturas, lo que demuestra que la formación en investigación permea positivamente los procesos académicos de otras actividades curriculares.

\section{REFERENCIAS BIBLIOGRÁFICAS}

BIBLIOGRAPHYÁlvarez, S., Pérez, A y Suárez, L. (2008). Hacia Un Enfoque De La Educación En Competencias. Consejería de Educación y Ciencia. https:// redined.mecd.gob.es/xmlui/bitstream/ handle/11162/2576/01720082000075. pdf?sequence $=1$

Bravo, N. (2007). Competencias Proyecto Tuning-Europa, Tuning-América Latina. Ministerio de Transportes y Comunicaciones y la wildlife I ecology I conservation [Archivo PDF]. https:// www.google.com/url?sa=t\&source $=w$ eb\&rct=j\&url=http://www.cca.org.mx/ profesores/cursos/hmfbcp_ut/pdfs/ $\mathrm{m} 1 /$ competencias_proyectotuning.pd f\&ved=2ahUKEwjSrb3j3KfwAhVbF1 kFHVJKBbgQFjAAegQIAxAC\&usg= AOvVawOfygEudHxtmN1gQcxuNvCM

Campos, J. y Chinchilla, A. (2009). Reflexiones acerca de los desafíos en la formación de competencias para la investigación en educación superior. Actualidades Investigativas en Educación, 9(2), 1 - 20. https://www.redalyc.org/ pdf/447/44713058023.pdf 
Contreras Ramírez Paris, J. V., Ramírez Paris Colmenares, X., \& Hernández Vergel, V. K. (2019). Factores que influyen en el desempeño escolar de los estudiantes de Básica Primaria de una institución educativa del área metropolitana de Cúcuta. Revista Perspectivas, 4(1), 6-13. https://doi. org/10.22463/25909215.1746

Cuevas, L., Guillén, D. M., \& Rocha, V. E. (2011). Las competencias en investigación como puentes cognitivos para un aprendizaje significativo. Razón y Palabra (77), 1 8. Recuperado de: https://www.redalyc. org/pdf/1995/199520010084.pdf

Espinoza, E. R. (2016). Formación de competencias investigativas en los estudiantes universitarios. Atenas. Revista Científico Pedagógica, 1 (33). 18 - 31. Recuperado de: http://atenas. umcc.cu/index.php/atenas/article/ $\begin{array}{llll}42 & 2 & \text { view/183/341 }\end{array}$

Figel, J. (2009). Competencias clave para el aprendizaje permanente. Altablero. Recuperado de https:// www.mineducacion.gov.co/1621/ article-210023.html

Gamboa, A. A., Vargas, R. y Hernández, C. A. (2017). Investigación formativa: pretensiones curriculares $y$ representaciones sociales de actores educativos en el programa de Enfermería de la Universidad Francisco de Paula Santander - Cúcuta, Colombia. Revista Universidad y Salud, 19(1), 6066. DOI: http://dx.doi.org/10.22267/ rus. 171901.69

Gamboa, A. (2016). Calidad de la educación superior: pretensiones y realidades institucionales. Bogotá, Colombia: Ecoe Ediciones
Granero, J. (2005). El Desarrollo de Competencias y la Elaboración de la Guía Docente en Enfermería MédicoQuirúrgica. Desarrollo Científ Enferm, 13(1), 10 - 15. Recuperado. de: http:// www.index-f.com/dce/13pdf/13-10.pdf

Gutiérrez, J. A. (2015). Modelo de competencias investigativas empresariales desde la Universidad, Empresa y Estado en Colombia. Praxis \& Saber, 6 (12), 241-267. Recuperado de: https://www.redalyc.org/articulo. oa?id=477247216012

Hernández-Suárez, C. A., Prada-Núñez, R., \& Avendaño-Castro, W. R. (2020). Escala para la valoración de las competencias de producción científica en docentes de Educación Superior. Revista Espacios, 41(44), 113-129. 10.48082/espaciosa20v41n44p09

Hernández-Suárez, C. A., Prada-Núñez, R., \& Gamboa-Suárez, A. A. (2020). Validación de una escala para evaluar competencias investigativas en docente de básica y media. Revista Boletín Redipe, 10(5). Recuperado de: https:// revista.redipe.org/index.php/1/article/ view/1335

Hernández, R., Fernández, C. y Baptista, P. (2014). Metodología de la Investigación Cientifica. México: McGraw Hill

López, G. E. (2016). En torno al concepto de competencia: un análisis de fuentes. Revista de Currículum y Formación de Profesorado, 20(1), 311-322. Recuperado de: https://www.redalyc. org/articulo.oa?id=56745576016 
Mulder, M. W. (2008). El concepto de competencia en el desarrollo de la educación y formación profesional en algunos Estados miembros de la UE: un análisis crítico. Profesorado. Revista de Currículum y Formación de Profesorado, 12(3), 1 - 26. Recuperado de: http:// www.ugr.es/ recfpro/rev123ART6.pdf

Reiban, R. E., De la Rosa, H., Zeballos, J. M. (2017). Competencias investigativas en la Educación Superior. Revista Publicando, 4 (10). 395 - 405. Recuperado de: https://core.ac.uk/ download/pdf/236644238.pdf

Ribes, I. E. (2011). El concepto de competencia: su pertinencia en el desarrollo psicológico y la educación. Bordón, 63(1), 33-45. Recuperado de: file:///C:/ Users/usuario/Downloads/DialnetEIConceptoDeCompetencia-3600075. pdf

Rivas, L. A. (2011). Las nueve competencias de un investigador. Investigación administrativa, $\quad 40(108), \quad 34$ - 54. Recuperado de: http:// www.scielo.org. mx/ scielo. php?script=sci_arttext\&pid=S2448$76782011000200034 \&$ Ing=es\&tIng=es.
Urbina-Cárdenas, J. E., Gamboa-Suárez, A. A., \& Prada-Núñez, R. (2020). Procesos formativos en investigación: relatos de estudiantes y profesores de un programa de trabajo social. Revista Espacios. 4(39), 22-31. Recuperado de: https://w.revistaespacios.com/ a20v41n39/a20v41n39p03.pdf

Villamizar-Acevedo, G. A., Lozano-León, S. G., \& Sierra-Garavito, E. D. (2017). Creencias sobre las fuentes y formas de acceso al conocimiento generadas en las prácticas pedagógicas desde la perspectiva del estudiante. Revista Perspectivas, 2(1), 18-27. https://doi. org/10.22463/25909215.1281 